\title{
Dual-Energy X-Ray Absorptiometry (DEXA) Scan Versus Computed Tomography for Bone Density Assessment
}

\author{
Malak Alawi ${ }^{1}$, Azra Begum ${ }^{1}$, Mohammed Harraz ${ }^{2}$, Hani Alawi ${ }^{3}$, Shahd Bamagos ${ }^{4}$, Abdulmalek \\ Yaghmour $^{1}$, Lubna Hafiz ${ }^{5}$ \\ 1. Radiodiagnosis, King Abdul Aziz Hospital, Makkah, SAU 2. Radiodiagnosis, Mansoura University Hospital, \\ Mansoura, EGY 3. Family Medicine, Primary Health Center Clinic, Makkah, SAU 4. Radiodiagnosis, Umm Al Qura \\ University, Makkah, SAU 5. Family Medicine, Al Zaher Primary Health Care Center, Makkah, SAU
}

Corresponding author: Azra Begum, drazrabegum@gmail.com

\section{Abstract \\ Rationale and objective}

Osteoporosis, a common non-pathological disease of bones, has been the cause of many disastrous consequences, in terms of physical, psychological, social, and economic loss. Therefore, it is crucial to diagnose it early for timely prevention and treatment of osteoporotic fractures. Dual-Energy X-Ray Absorptiometry (DEXA) is currently routinely used for determining bone mineral density. However, it has its limitations. Nowadays, CT technology has advanced so rapidly that the Hounsfield units (HU) values can be used in opportunistic screening for osteoporosis in patients during routine CT abdomen for other causes. Hence, there would be no need for additional study with DEXA and also reduce radiation exposure. The aim of our research is to determine whether there is a correlation between the bone mineral density and the Tscore measured by DEXA and the HU values measured from the diagnostic CT images of L1-4 vertebrae. Also, to determine reference CT values that would help in screening the patients with osteoporosis.

\section{Materials and methods}

We conducted a retrospective study of 78 female patients who underwent CT lumbar spine, abdomen, and pelvis in our hospital between the years 2016-2020. We collected data of patients who performed DEXA and CT scans within an interval of up to two years. The final collected data was analyzed to find correlation values of HU with age group and with DEXA bone mineral density (BMD) and T-score using Pearson correlation coefficient.

\section{Results}

The mean of the 78 patients was 61.1 (range 37-88 years). Mean HU values decreased consistently with age, from 202.17 HU in the fifth decade to $71 \mathrm{HU}$ in the ninth decade. Average L1-4 HU values ranged from $71 \mathrm{HU}$ to $202.17 \mathrm{HU}$ (mean with standard deviation), while their T-score ranged from -4.4 to +2.4 (mean was $1.7 \pm 1.41$ ), and their BMD ranged from 0.62 to $1.465 \mathrm{~g} / \mathrm{cm}^{2}$ (mean, $0.974 \pm 0.175 \mathrm{~g} / \mathrm{cm}^{2}$ ). For each lumbar vertebra, the correlations of HU values with bone mineral density and T-score were calculated separately.

Review began 01/20/2021 Review ended 02/03/2021 Published 02/10/2021

\section{(c) Copyright 2021}

Alawi et al. This is an open access article distributed under the terms of the Creative Commons Attribution License CC-BY 4.0., which permits unrestricted use, distribution, and reproduction in any medium, provided the original author and source are credited.
For L1-4 vertebrae, the correlation coefficients $\left(\mathrm{r}^{2}\right)$ for the HU value and T-score were $0.544,0.600,0.611$, and 0.600 , respectively. The correlation coefficients $\left(\mathrm{r}^{2}\right)$ for the HU value and bone mineral density were $0.581,0.623,0.653,0.612$, respectively. All the calculated correlations were significant $(\mathrm{p}<0.001)$. Therefore, it was concluded that there was a positive correlation between the HU values and the DEXA for the BMD and between the HU values and the T-score. Based on the WHO guidelines, the T-scores of the lumbar vertebrae were classified into three groups. The mean HU values for the subjects in the normal group were 174.05 (95\% confidence interval, 153-194.49), in the osteopenia group were $120.45 \mathrm{HU}$ (95\% confidence interval, 106.98133.91), and in the osteoporosis group were $115 \mathrm{HU}$ (95\% confidence interval, 104.60-125.40). The differences in the mean $\mathrm{HU}$ values between the groups were significant.

\section{Conclusion}

On analyzing the results of our study, we reached the conclusion that there is a positive correlation between the HU calculated from CT with automated exposure control and BMD calculated from the DEXA. Thus CT scans done for various reasons, for example, the abdomen, lumbar spine, etc. can provide us with information about the patient's bone density as well. CT is a very popular, easily accessible, reproducible, and reliable tool for measuring HU values and thereby in the opportunistic screening of osteoporosis.

Categories: Radiology

Keywords: dexa, ct, bone density, t-score, bmd, bone mineral density, correlation, osteoporosis, osteopenia, who 


\section{Introduction}

Osteoporosis is a disease of the bones in which low bone mass and structural deterioration of bone tissue lead to an increase in bone fragility. It is by far the most common metabolic bone disease which has affected over 200 million people all over the world. It has also been the cause of many disastrous consequences, in terms of physical, psychological, social, and economic loss. The unfortunate reason for this is that it is often overlooked and under-treated because it does not show any clinical symptoms until it suddenly manifests as a fracture. Therefore it is very crucial to diagnose it early for timely prevention and treatment of and osteoporotic fractures and their unfortunate consequences $[1,2,3]$.

Osteoporosis is monitored using the measure of bone mineral density (BMD) as it is directly related to bone strength [4]. BMD measurements are classified according to the T-score as specified by the World Health Organization (WHO) in 1994 [5]. A T-score is the standard deviation of the BMD of an individual patient compared with a young, healthy reference population, matched for sex and ethnicity. A T-score that lies between the values of -2.5 and -1 is defined as osteopenia while a T-score that is equal to or less than -2.5 is defined as osteoporosis. Initially, this definition was intended to be used in postmenopausal women. Later, the International Society for Clinical Densitometry (ISCD) modified and used this definition to classify BMD in pre-and post-menopausal women, men, and children by using Dual-Energy X-ray Absorptiometry (DEXA), which calculated the BMD (grams per square centimeter) as well as the Z-scores and T-scores. Zscores are standard deviations compared with an age-matched reference population, while T-scores are standard deviations compared with a young adult reference population [6].

Dual-energy X-ray Absorptiometry (DEXA) and quantitative computed tomography (QCT) of the lumbar spine were considered as preferred methods for the evaluation of BMD. DEXA was recommended by the World Health Organization (WHO) as a gold standard for diagnosing osteoporosis. Because of its availability, relatively minimal radiation exposure, and simplicity of use, DEXA is the most commonly employed quantitative radiologic method to assess bone mass [7].

However, DEXA also has disadvantages that need to be kept in mind:

1) It is a two-dimensional measurement, which only measures density/area (in grams per square centimeter) and not the volumetric density (in milligrams per cubic centimeter) such as with quantitative computed tomography (CT).

2) Areal BMD is susceptible to bone size and will thus overestimate fracture risk in individuals with a small body frame, who will have lower areal BMD than normal-sized individuals.

3) Spine and hip DEXA are also sensitive to degenerative changes, and individuals with substantial degenerative disease will have increased areal density, which will suggest a lower fracture risk than is actually present. All structures overlying the spine, such as aortic calcifications, or morphologic abnormalities, such as after laminectomy at the spine, will affect BMD measurements; it is also critical to check DEXA images for artifacts, which may alter BMD values [6].

Another tool to measure BMD is using quantitative computed tomography (QCT). This idea was suggested in the 1970s [7]. In the initial days, CT technology was not very well developed as it took a long time and there was more exposure to radiation. Therefore, it was not considered. However, now CT technology has made rapid advancement, making it a promising tool in measuring BMD [8].

The Hounsfield Unit (HU) values can be a very useful index to opportunistically screen patients who undergo routine CT abdomen and pelvis for other reasons and thus obviate the need to do another test like DEXA for BMD assessment. There will also be a reduction in radiation dose.

\section{Aims and objectives}

Our study was aimed at finding out whether there was a correlation between the bone mineral density and the T-scores measured by DEXA and the HU values measured from the diagnostic CT images of L1-L4 vertebrae. We also aimed to determine reference CT values that would help in screening patients with osteoporosis.

\section{Materials And Methods}

We conducted a retrospective study of 78 female patients, of both pre- and postmenopausal age groups and irrespective of risk factor status, who underwent CT lumbar spine, abdomen, and pelvis in our hospital between the years 2016-2020. Data of patients who underwent DEXA and CT scans within an interval of up to two years was collected. The patients with extensive spine degenerative changes or post-surgical spine intervention, etc., were excluded from our study. The final collected data was analyzed to find correlation values of HU with age group and with DEXA bone mineral density and T-score using Pearson correlation coefficient. 


\section{Cureus}

All imaging was performed by a 64-slice multidetector CT (MDCT) scanner (Discover, GE Healthcare, Chicago USA). The CT parameters included a slice thickness of $1.0 \mathrm{~mm}$, a tube voltage of $120 \mathrm{kV}$, a tube current of $330 \mathrm{~mA}$ (DoseRight automated exposure control system), and a bone reconstruction algorithm (window width/window level, 2050/250). Two-dimensional reconstructions were obtained in the coronal and sagittal planes.

DEXA scans were performed with the use of a Lunar Bone Densitometer (GE Healthcare). The T-scores and the bone mineral density (grams per square centimeter) were obtained from the DEXA scan for the L1 to L4 lumbar vertebrae.

The HU values were calculated by using a picture archiving and communication system (PACS; GE Digital Healthcare). An elliptical region of interest was placed over the axial CT image of the lumbar vertebrae (L1 till L4) excluding the cortices. A protocol described by Pickhardt et al. [9], was used to measure the HU for each vertebra (Figure 1).

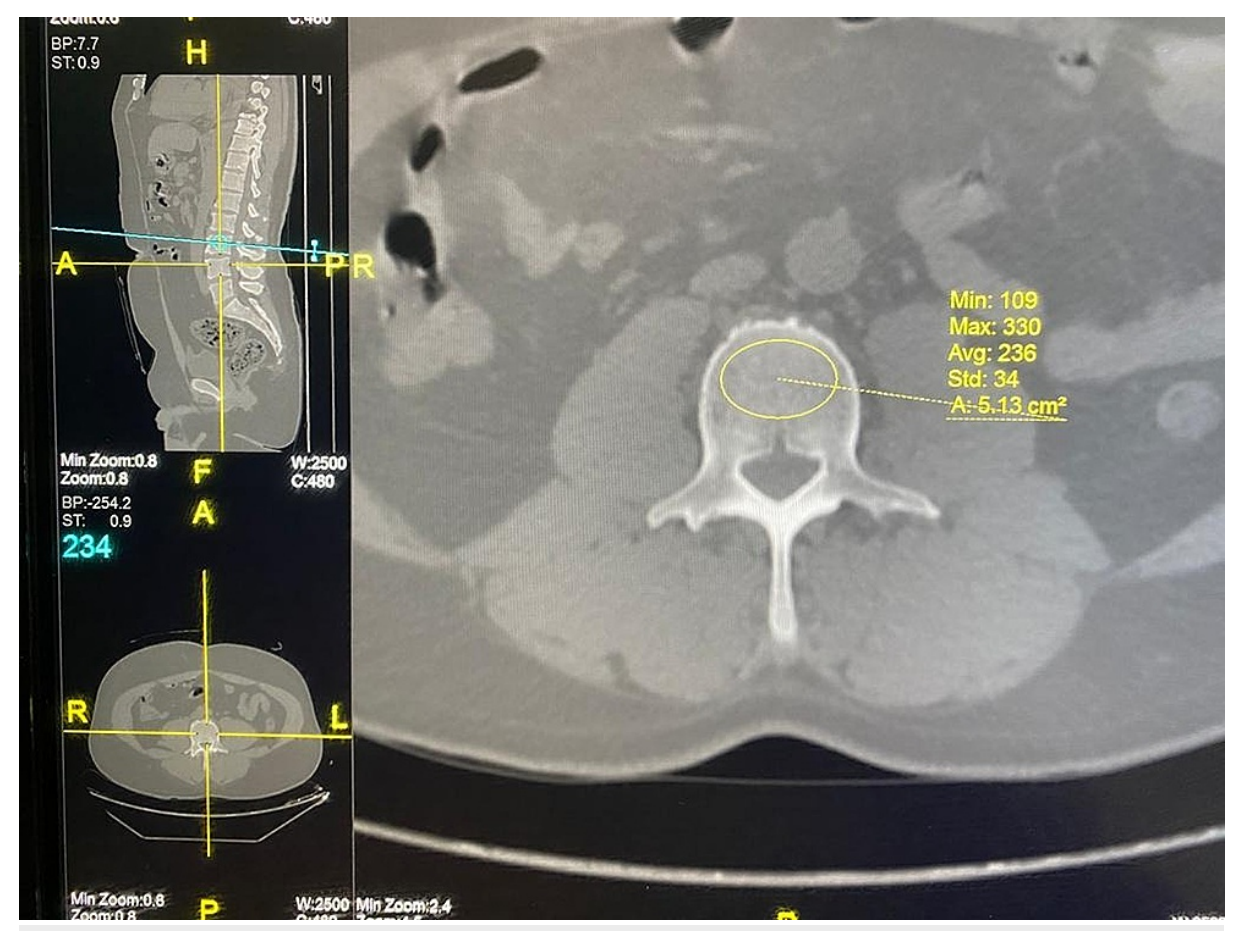

FIGURE 1: Method to measure HU value on CT image for each vertebra

Axial CT images of lumbar vertebrae illustrating the method of determining HU values by using the PACS system. Multi-planar reconstructed images (A) an oval region of interest (ROI) is placed over the axial plane in the mid vertebra level.

PACS: picture archiving and communication system

\section{Statistical analysis}

The Statistical Analysis was carried out using Statistical Package for Social Sciences (SPSS) software, Version 22.0 (IBM Corp., Armonk, NY, USA). The correlation parameters between DEXA and CT were calculated for $\mathrm{HU}$ values of each vertebral level, L1 till L4. All statistical tests were two-sided and performed at a significant level of $\mathrm{p}<0.05$. Correlation values of $\mathrm{HU}$ with age group and with DEXA bone mineral density and T-score using Pearson correlation coefficient (0.0-0.19 -very weak correlation, 0.20-0.39 - weak correlation, 0.40-0.59 - moderate correlation, 0.60-0.79 - strong correlation, 0.80-1.0 - very strong correlation). Mean and $95 \%$ confidence intervals were calculated of patients with bone density divided into three groups - normal, osteopenia, and osteoporosis - as per WHO guidelines.

\section{Results}

The mean age of the 78 patients was 61.1 years (range $37-88$ years). It was observed that the mean $\mathrm{HU}$ values showed a tendency to decrease with age (Table 1, Figure 2). 


\section{Cureus}

\begin{tabular}{|c|c|c|c|c|c|c|}
\hline \multirow{2}{*}{$\begin{array}{l}\text { Age } \\
\text { (Years) }\end{array}$} & \multirow{2}{*}{$\begin{array}{l}\text { Patient } \\
\text { No. }\end{array}$} & \multicolumn{4}{|c|}{ Hounsfield Units (Means \pm Standard Deviation) } & \multirow{2}{*}{ Average } \\
\hline & & L1 & L2 & L3 & L4 & \\
\hline 40 to 49 & 3 & $\begin{array}{l}191.3333 \pm \\
21.45538\end{array}$ & $206 \pm 4.358899$ & $\begin{array}{l}216.3333 \pm \\
31.65965\end{array}$ & $195 \pm 16.522 / 1$ & $\begin{array}{l}202.1667 \pm \\
3.826988\end{array}$ \\
\hline 50 to 59 & 35 & $\begin{array}{l}145.4286 \pm \\
41.12555\end{array}$ & $\begin{array}{l}145.6857 \pm \\
45.01031\end{array}$ & $\begin{array}{l}136.3429 \pm \\
48.57374\end{array}$ & $135 \pm 49.8 / 101$ & $\begin{array}{l}140.6143 \pm \\
42.37192\end{array}$ \\
\hline 60 to 69 & 18 & $\begin{array}{l}112.1111 \pm \\
31.32165\end{array}$ & $\begin{array}{l}107.3333 \pm \\
28.77090\end{array}$ & $\begin{array}{l}96.11111 \pm \\
28.51189\end{array}$ & $96.8333 \pm 24.39684$ & $\begin{array}{l}103.0972 \pm \\
25.90553\end{array}$ \\
\hline 10 to 19 & 14 & $\begin{array}{l}104.3571 \pm \\
28.78997\end{array}$ & $\begin{array}{l}91.78571 \pm \\
30.40035\end{array}$ & $\begin{array}{l}102.2143 \pm \\
34.53466\end{array}$ & $\begin{array}{l}92.78571 \pm \\
26.46094\end{array}$ & $\begin{array}{l}97.78571 \pm \\
24.23282\end{array}$ \\
\hline $\begin{array}{l}\text { Over \& = } \\
80\end{array}$ & 4 & $113.75 \pm 46.28445$ & $51 \pm 24.385 / 9$ & $59 \pm 36.1755$ & $60.25 \pm 51.93826$ & $71 \pm 29.62333$ \\
\hline
\end{tabular}

\section{TABLE 1: Hounsfield Unit values of lumbar vertebrae versus age}

Data of Hounsfield unit values obtained from lumbar computed tomography scans arranged according to decades of life.

$2 a$
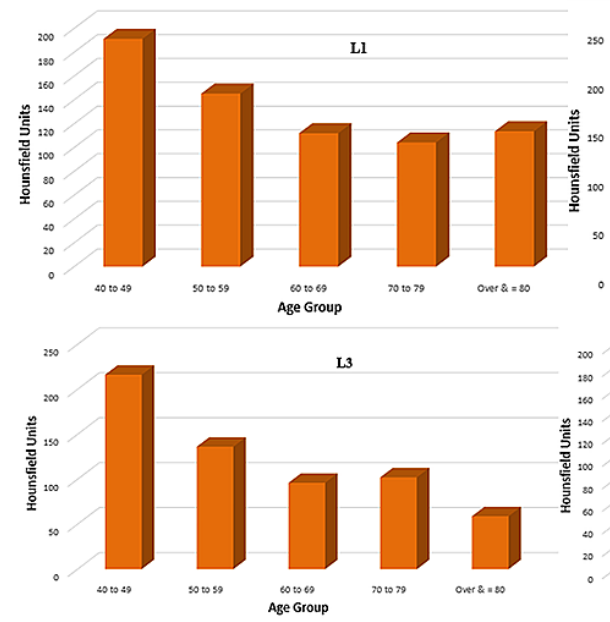

$2 c$
$2 b$

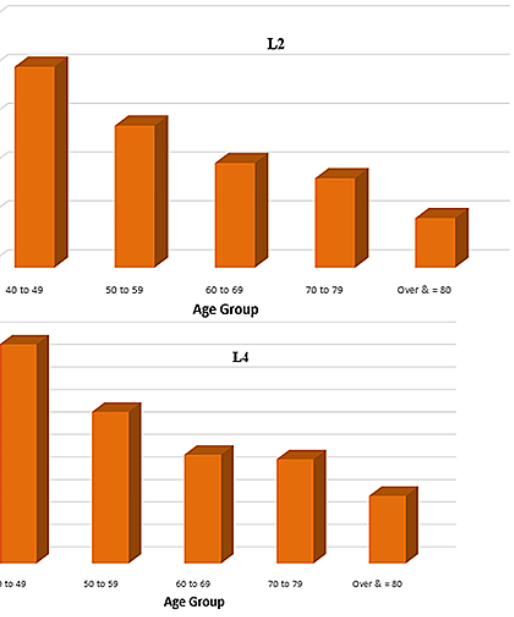

$2 \mathrm{~d}$

\section{FIGURE 2: Hounsfield Unit values on CT of lumbar vertebrae versus age}

Mean Hounsfield unit values among decadal age groups decreases as age increases. 2a - L1 vertebra HU values versus age groups; $2 b$ - L2 vertebra $\mathrm{HU}$ values versus age groups; $2 \mathrm{c}$ - L3 vertebra HU values versus age groups; $2 \mathrm{~d}$ - L4 vertebra $\mathrm{HU}$ values versus age groups.

Average L1-4 HU values ranged from $71 \mathrm{HU}$ to $202.17 \mathrm{HU}$ (mean with standard deviation), while their Tscore ranged from -4.4 to +2.4 (mean was $-1.7 \pm 1.41$ ), and their bone mineral density ranged from 0.62 to $1.465 \mathrm{~g} / \mathrm{cm} 2$ (mean $0.974 \pm 0.175 \mathrm{~g} / \mathrm{cm} 2$ ). For each lumbar vertebra, the correlations of HU values with bone mineral density and T-score were calculated separately. For L1-4 vertebrae, the correlation coefficients $\left(\mathrm{r}^{2}\right)$ for the $\mathrm{HU}$ value and $\mathrm{T}$-score were $0.544,0.600,0.611$, and 0.600 , respectively (Figure 3 ). The correlation coefficients $\left(\mathrm{r}^{2}\right)$ for the $\mathrm{HU}$ value and bone mineral density were $0.581,0.623,0.653$, and 0.612 , respectively (Figure 4). All the calculated correlations were significant ( $\mathrm{p}<0.001)$. Therefore, it was concluded that there was a positive correlation between the HU values and the DEXA for the BMD and between the HU values and the T-score. 


\section{Cureus}
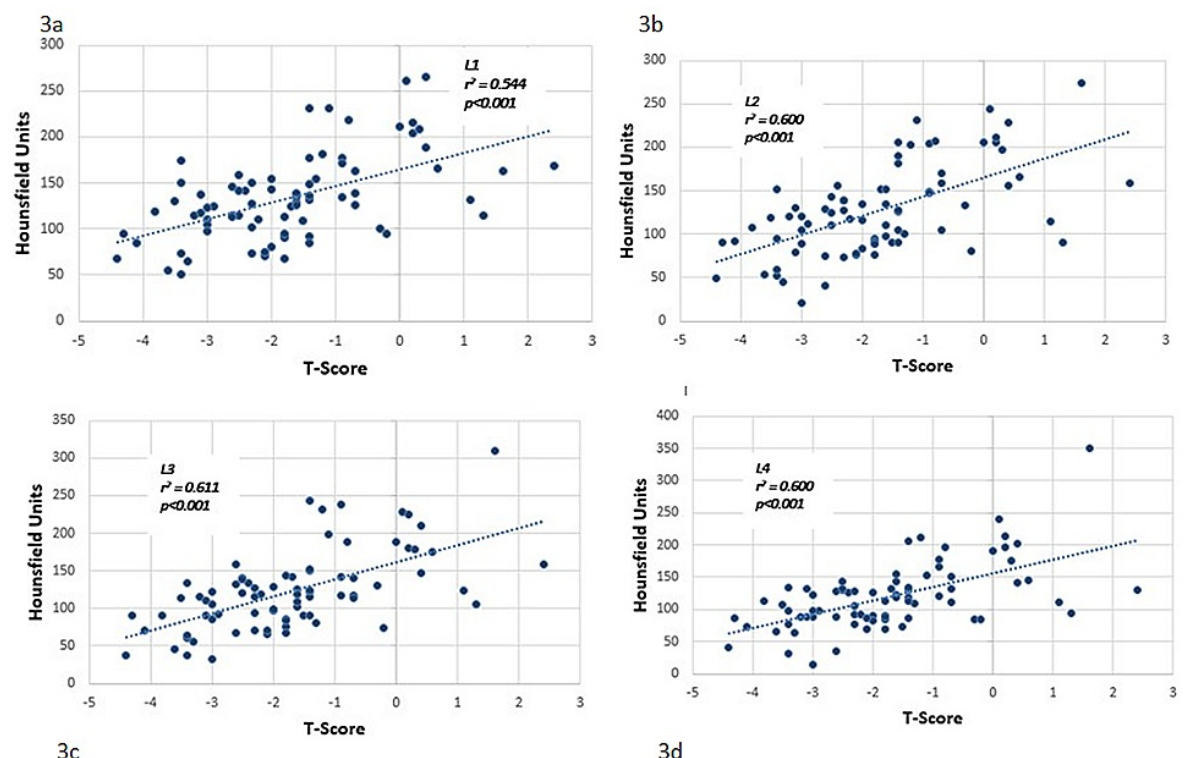

FIGURE 3: Scatter plot showing the correlation between Hounsfield unit values from CT and T-scores form Dual-Energy X-Ray Absorptiometry

Scatter plot showing correlations between Hounsfield unit values obtained from CT and T-scores obtained from Dual-Energy X-Ray Absorptiometry for lumbar vertebrae: 3a - L1, 3b - L2, 3c - L3, 3d - L4. It showed significant correlation coefficients $(p<0.001)$.

$4 a$

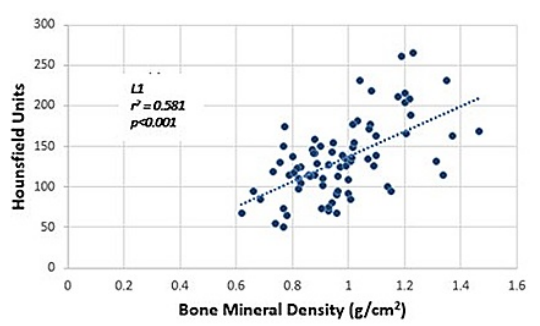

$4 \mathrm{c}$

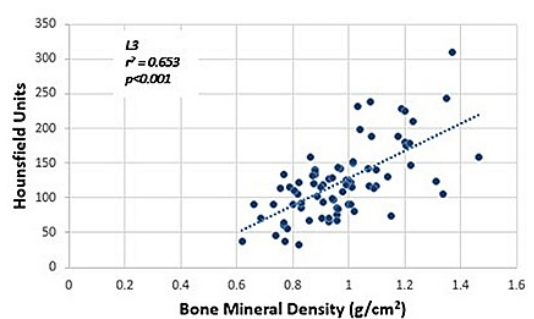

$4 \mathrm{~b}$

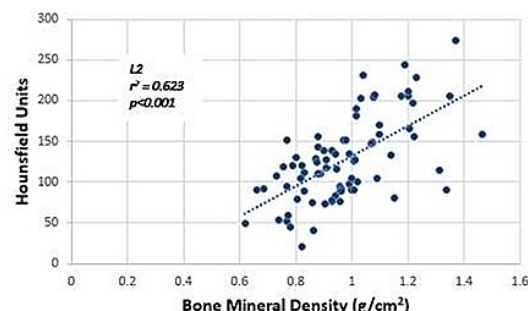

$4 d$

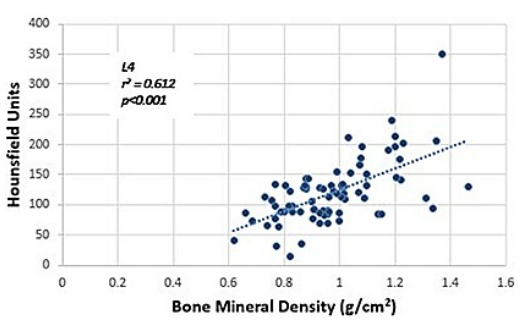

FIGURE 4: Scatter plots showing the correlation between Hounsfield unit values obtained from CT scans and bone mineral density obtained from Dual-Energy X-Ray Absorptiometry

Scatter plots showing correlations between Hounsfield Unit values obtained from CT scans and bone mineral density obtained from Dual-Energy X-Ray Absorptiometry for lumbar vertebrae: 4a - L1, 4b - L2, 4c - L3, 4d L4. It showed significant correlation coefficients $(p<0.001)$.

According to the WHO guidelines, the T-scores of the lumbar vertebrae were classified into normal (-1.0 or greater), osteopenia (less than -1.0 and greater than -2.5 ), and osteoporosis ( -2.5 or less). The mean HU values for the subjects in the normal group were 174.05 (95\% confidence interval, 153-194.49), in the osteopenia group were $120.45 \mathrm{HU}$ (95\% confidence interval, 106.98 - 133.91), and in the osteoporosis group 
were $115 \mathrm{HU}$ (95\% confidence interval, 104.60-125.40) (Table 2). There was no significant difference in the mean HU between the osteopenia and osteoporosis groups. However, the difference in the mean HU values was significant between the normal group and osteopenia $(\mathrm{p}<0.05)$.

\begin{tabular}{|c|c|c|c|}
\hline & \multirow{2}{*}{ T-Score } & \multicolumn{2}{|c|}{ Hounsfield Units } \\
\hline & & Mean and SD & $95 \%$ Confidence Interval \\
\hline Normal & $\geq-1.0$ & $174.057 \pm 46.08$ & $153.63-194.49$ \\
\hline Osteopenia & -1.0 & $120.45 \pm 37.34$ & $106.98-133.91$ \\
\hline Osteoporotic & $-2.5 \leq$ & $115 \pm 41.30$ & $104.60-125.40$ \\
\hline
\end{tabular}

TABLE 2: Mean and $95 \%$ confidence intervals of patients with bone density divided into three groups-normal, osteopenia and osteoporosis

\section{Discussion}

On observation and analysis of the data, it was seen that there is a moderate correlation between the HU values obtained from CT and the bone mineral density and T-score determined by DEXA. This could probably be due to the fact that DEXA scan calculations include the cortex and the posterior elements, while only the trabecular portion of the vertebra was used to determine the HU value on CT.

There are a few studies which have shown the possibility of using diagnostic CT images (with automated exposure control) for estimating bone mineral density [10-13].

DEXA is currently routinely used for determining bone mineral density, because of the advantage that it is easily available, easy to perform, and has a low radiation dose (0.009-0.027 mSv). While radiation dose of Computed Tomography (CT) is higher (0.06-2.5 mSv) [14].

Previously, quantitative CT required the use of a calibration phantom with a known density. The phantom was scanned with the patient to convert HU values into bone mineral density and to permit calibration of other factors that may interfere with the results [7]. With the advent of automated exposure control on modern CT scanners, the calibrating phantoms that were historically used for quantitative CT are not required [10].

Our study showed that there was a moderate correlation between HU values and T-score. Therefore, patients who have decreased bone density can be identified easily during routine CT scans done for various reasons and directed to DEXA imaging, which can help to determine the category of bone density - normal, osteopenia, or osteoporosis. Thus, CT can be used as an opportunistic screening tool for decreased bone density.

The results of our study were similar to the previous studies which support the use of CT in screening patients for decreased bone density. We suggest a reference value of $150 \mathrm{HU}$ on CT imaging as a cut-off for determining patients with low bone density. Our reference value is corroborating with the previous studies $[9,15,16]$.

As stated earlier, the radiation dose of CT is higher than DEXA. Therefore, though CT may not be recommended as the standard for bone density measurement, it can still be used as an opportunistic tool in patients undergoing routine CT scans for other reasons to detect those with low bone density and who can be referred for DEXA imaging and further treatment.

The limitations of our study are that our measurements and calculations were done on a single type of CT scanner and DEXA machine. We do not know the reproducibility of the results in other different types of CT and DEXA scanners. Another limitation is that the number of patients was limited and hence the need for a large population to determine the accuracy and reliability of the results. The third limitation was the gap of one to two years between CT and DEXA imaging may alter the results. Also, the HU values were calculated from trabeculated bone on CT, while DEXA values are calculated including the cortical bone with the trabeculated bone, which can cause a difference in correlation of both CT and DEXA.

\section{Conclusions}

On analyzing the results of our study, we reached the conclusion that there is a positive correlation between the HU calculated from CT with automated exposure control and BMD calculated from the DEXA. The 
determination of reference cut off value of $\mathrm{HU}$ can be applied in all routine CT scans for detecting patients with low bone density. Thus, CT being a very popular, easily accessible, reproducible, and reliable tool for measuring $\mathrm{HU}$ values, can thereby be useful in the opportunistic screening of patients with decreased bone density, who can be referred for DEXA and subsequent management.

\section{Additional Information \\ Disclosures}

Human subjects: Consent was obtained or waived by all participants in this study. Institutional Review Board, Ministry of Health, Makkah issued approval H-02-K-076-1220-427. This is to inform you that the above mentioned proposal has been reviewed and discussed by IRB Committee and was approved according to the ICH GCP guidelines. Animal subjects: All authors have confirmed that this study did not involve animal subjects or tissue. Conflicts of interest: In compliance with the ICMJE uniform disclosure form, all authors declare the following: Payment/services info: All authors have declared that no financial support was received from any organization for the submitted work. Financial relationships: All authors have declared that they have no financial relationships at present or within the previous three years with any organizations that might have an interest in the submitted work. Other relationships: All authors have declared that there are no other relationships or activities that could appear to have influenced the submitted work.

\section{Acknowledgements}

We are grateful to Ms. Mashael Faisal Al Yamani and Ms. Reem Bandar Al Juaid for their help in file submission, editing, and typing of the article.

\section{References}

1. Porter JL, Varacallo M: Osteoporosis. StatPearls [Internet]. StatPearls Publishing, Treasure Island; 2020.

2. Cooper C, Campion G, Melton LJ: Hip fractures in the elderly: a world-wide projection . Osteoporosis Int. 1992, 2:285-289. 10.1007/BF01623184

3. Osteoporosis: practice essentials, background, pathophysiology. (2021). Accessed: January 20, 2021: http://emedicine.medscape.com/article/330598-overview.

4. Mao SS, Li D, Luo Y, Syed YS, Budoff MJ: Application of quantitative computed tomography for assessment of trabecular bone mineral density, microarchitecture and mechanical property. Clin Imaging. 2016, 40:330338. 10.1016/j.clinimag.2015.09.016

5. World Health Organization: Assessment of Fracture Risk and Its Application to Screening for Postmenopausal Osteoporosis: Report of a WHO Study Group [Meeting Held in Rome from 22 to 25 June 1992]. World Health Organization, Geneva; 1994.

6. Link TM: Osteoporosis imaging: state of the art and advanced imaging . Radiology. 2012, 263:3-17. 10.1148/radiol.12110462

7. Adams JE: Quantitative computed tomography. Eur J Radiol. 2009, 71:415-424. 10.1016/j.ejrad.2009.04.074

8. Goo HW: CT radiation dose optimization and estimation: an update for radiologists . Korean J Radiol. 2012, 13:1-11. 10.3348/kjr.2012.13.1.1

9. Pickhardt PJ, Lee LJ, del Rio AM, et al.: Simultaneous screening for osteoporosis at CT colonography: bone mineral density assessment using MDCT attenuation techniques compared with the DXA reference standard. J Bone Miner Res. 2011, 26:2194-2203. 10.1002/jbmr.428

10. Link TM, Koppers BB, Licht T, Bauer J, Lu Y, Rummeny EJ: In vitro and in vivo spiral CT to determine bone mineral density: initial experience in patients at risk for osteoporosis. Radiology. 2004 , 231:805-811. 10.1148/radiol.2313030325

11. Miyabara Y, Holmes D 3rd, Camp J, Miller VM, Kearns AE: Comparison of calibrated and uncalibrated bone mineral density by CT to DEXA in menopausal women. Climacteric. 2012, 15:374-381. 10.3109/13697137.2011.618566

12. Papadakis AE, Karantanas, AH, Papadokostakis G, Damilakis J: Assessment of the morpho-densitometric parameters of the lumbar pedicles in osteoporotic and control women undergoing routine abdominal MDCT examinations. J Bone Miner Metab. 2011, 29:352-358. 10.1007/s00774-010-0227-7

13. Papadakis AE, Karantanas AH, Papadokostakis G, Petinellis E, Damilakis J: Can abdominal multi-detector CT diagnose spinal osteoporosis?. Eur Radiol. 2009, 19:172-176. 10.1007/s00330-008-1099-2

14. Damilakis J, Adams JE, Guglielmi G, Link TM: Radiation exposure in X-ray-based imaging techniques used in osteoporosis. Eur Radiol. 2010, 20:2707-2714. 10.1007/s00330-010-1845-0

15. Lee S, Chung CK, Oh SH, Park SB: Correlation between bone mineral density measured by dual-energy Xray absorptiometry and Hounsefield units measured by diagnostic CT in lumbar spine. J Korean Neurosurg Soc. 2013, 54:384-389. 10.3340/jkns.2013.54.5.384

16. Islamian JP, Garoosi I, Fard KA, Abdollahi MR: Comparison between the MDCT and the DXA scanners in the evaluation of BMD in the lumbar spine densitometry. Egypt J Radiol Nucl Med. 2016, 47:961-967.

10.1016/j.ejrnm.2016.04.005 